EPJ Web of Conferences 58, 01016 (2013)

DOI: $10.1051 /$ epjconf/20135801016

(C) Owned by the authors, published by EDP Sciences, 2013

\title{
Could quantum decoherence and measurement be deterministic phenomena?
}

\author{
Jean-Marc Sparenberg ${ }^{1, a}$, Réda Nour ${ }^{1}$, and Aylin Manço ${ }^{1}$ \\ ${ }^{1}$ Université libre de Bruxelles (ULB), École polytechnique de Bruxelles, \\ Nuclear Physics and Quantum Physics, CP 229, B 1050 Brussels, Belgium
}

\begin{abstract}
The apparent random outcome of a quantum measurement is conjectured to be fundamentally determined by the microscopic state of the macroscopic measurement apparatus. The apparatus state thus plays the role of a hidden variable which, in contrast with variables characterizing the measured microscopic system, is shown to lead to a violation of Bell's inequalities and to agree with standard quantum mechanics. An explicit realization of this interpretation is explored (for details, see [1]) for a primitive model of cloud chamber inspired by Mott [2]. Being highly non local, this interpretation of quantum mechanics is argued to open the way to faster-than-light information transfer.
\end{abstract}

\section{Introduction: advocacy for determinism}

The most disturbing feature of quantum physics is probably the fundamental randomness of the outcome of a quantum measurement: the repeated measurement of the same observable on a microscopic system prepared in the same state, with the same apparatus, can lead to different results. Think for instance about the detection of an $s$-wave $\alpha$-particle in a cloud chamber. Though the wave function is spherical, the observed individual tracks are linear, spread isotropically in apparently totally random directions. This seemingly unavoidable lack of determinism deeply bothered Einstein himself, as expressed by his famous opinion that "[God] does not throw dice" [3]. Indeed, determinism is one of the keystones of modern science, as experiments are only reproducible provided the same cause always produces the same effect. Giving up this very principle is in a sense equivalent to giving up science itself! Another motivation for not giving up determinism is timeless theories. In these, time does not have a fundamental character but rather emerges as a secondary quantity (see in particular the contribution by Barbour at this conference and [4]). In a deterministic world, the present moment is unambiguously related to the past from which it results and to the future which it determines, hence strengthening the idea that past, present and future are one and the same.

A possible way to recover determinism in quantum mechanics is to assume, in the spirit of Einstein, Podolsky and Rosen [5], that the knowledge of a microscopic system provided by quantum mechanics is incomplete. Hidden variables should exist, that determine the measurement outcome, even though they might not be accessible to the experimenter. Another fundamental advantage of hidden variables in Einstein's view is that they avoid violation of special relativity by quantum mechanics: instantaneous correlations between distant particles in an intricated state can be explained by

ae-mail: jmspar@ulb.ac.be

This is an Open Access article distributed under the terms of the Creative Commons Attribution License 2.0, which permits unrestricted use, distribution, and reproduction in any medium, provided the original work is properly cited. 
a common variable shared by the particles when they first interacted. The very logical assumption of hidden variables was however proved incorrect. Bell [6] and others [7-9] established inequalities that should be satisfied by measurement outcomes on intricated particles if such hidden variables existed, and that are violated by quantum mechanics predictions. Aspect and others [10-12] then checked experimentally that quantum predictions were correct, which ruled out hidden variables at least in their simplest form. These experiments also prove that quantum mechanics is highly non local and that the measurement performed on one particle indeed has an instantaneous impact on the measurement performed on another particle intricated with the first one, even at very large distances. The lack of determinism of quantum measurements then comes as a "savior" of special relativity [13]: since the outcome of the measurement on the first particle is random, the intrication between both particles, though it exists and is instantaneous, cannot be used to transmit information faster than light.

In the present work, we try to answer the question: what actually determines the result of a given quantum measurement? In the case of the $\alpha$-particle detection, what actually determines the direction of an observed track? We explore the hypothesis that hidden variables are not variables characterizing the microscopic system under study but rather the macroscopic apparatus (or apparatuses) used to study it. Indeed, quantum randomness always occurs when a microscopic system, well described by its wave function, interacts with a highly complex and unstable macroscopic system. Describing the state of such a complex system at the microscopic level is of course very difficult as its internal degrees of freedom (or more generally its "environment") cannot be monitored and appear as totally random to our "macroscopic eyes". It is however tempting to assume, as also suggested by Gaspard [14], that it is precisely this microscopic state of the system which is at the origin of the randomness observed during the measurement process. Our approach is thus related to the concept of decoherence [15-17]: there also, it is the interaction with a macroscopic system, e.g. a measurement apparatus, that makes a microscopic system initially in a linear superposition of states evolve into a standard statistical mixture of possible outcomes. However, decoherence is usually mathematically described by ad hoc terms in a master equation, with no attempt to explain particular outcomes of the decoherence process into one or another result. In the present approach, we go a step further, similarly to van Kampen [18], Gaspard and Nagaoka [19]: the internal state of the macroscopic system precisely determines which outcome will occur, not only the mere fact that probabilistically-acceptable outcomes do occur.

The aim of the present work is to test whether this simple deterministic interpretation leads to fundamental principle impossibilities or not. We first show that it leads to violations of Bell's inequalities, in agreement with standard quantum mechanics and with experimental results. Next we come back to the problem of $\alpha$-ray tracks in a cloud chamber. We explore the hypothesis that the positions of the atoms of the chamber actually determine the direction of the observed track. Finally, the price to pay for this interpretation is shown to be a strong non locality, which is not saved any more by quantum randomness [20], and which is speculated to open the way to faster-than-light communications.

\section{Apparatus hidden variables and Bell's inequalities}

Let us first recall the principle of the Einstein-Podolsky-Rosen thought experiment [5], as formulated by Bohm and Aharonov [7]. A system of two particles, 1 and 2, both with spin $\frac{1}{2}$ denoted as $s_{1}$ and $s_{2}$, is created in the maximally entangled singlet (i.e. spin 0 ) state

$$
|00\rangle=\frac{1}{\sqrt{2}}\left[|+\rangle_{\hat{\boldsymbol{u}} 1}|-\rangle_{\hat{\boldsymbol{u}} 2}-|-\rangle_{\hat{\boldsymbol{u}} 1}|+\rangle_{\hat{\boldsymbol{u}} 2}\right], \quad \forall \hat{\boldsymbol{u}},
$$

where $\hat{\boldsymbol{u}}$ is an arbitrary unit vector. The particles are assumed to stay in that spin state while moving away from each other. The spin of particle 1 is then measured along direction $\hat{\boldsymbol{a}}$ while the spin of particle 2 is measured along direction $\hat{\boldsymbol{b}}$, each measurement giving $+\frac{\hbar}{2}$ or $-\frac{\hbar}{2}$. 
Following Bell [6], we define the mean correlation between spin 1 and 2 measured along directions $\hat{\boldsymbol{a}}$ and $\hat{\boldsymbol{b}}$ as

$$
E(\hat{\boldsymbol{a}}, \hat{\boldsymbol{b}}) \equiv \frac{4}{\hbar^{2}}\left\langle\left(\boldsymbol{s}_{1} \cdot \hat{\boldsymbol{a}}\right)\left(\boldsymbol{s}_{2} \cdot \hat{\boldsymbol{b}}\right)\right\rangle .
$$

For the singlet state (1), this quantity takes the value

$$
E_{Q}(\hat{\boldsymbol{a}}, \hat{\boldsymbol{b}})=-\hat{\boldsymbol{a}} \cdot \hat{\boldsymbol{b}}
$$

in standard quantum mechanics. In particular, when $\hat{\boldsymbol{a}}=\hat{\boldsymbol{b}}$, the correlation between both measurements is perfect and one has $E_{Q}(\hat{\boldsymbol{a}}, \hat{\boldsymbol{a}})=-1$, whatever the distance between both particles at the time their spins are measured. This non-local correlation, typical of entangled quantum states, chagrined Einstein, hence leading to the hypothesis that the state of the particle pair was given not only by the wave function (1) but also by some hidden variable $\lambda$. This variable is assumed to be randomly distributed; its value is supposed to determine the result of any of the measurements. It thus also determines the value of correlation (3), which we now denote as $E_{\lambda}(\hat{\boldsymbol{a}}, \hat{\boldsymbol{b}})$. Such a variable avoids "spooky actions at a distance" apparently implied by state (1). However, Bell [6] brilliantly proved that the very existence of variable $\lambda$ leads to inequalities for the correlations calculated for three arbitrary directions $\hat{\boldsymbol{a}}, \hat{\boldsymbol{b}}, \hat{\boldsymbol{c}}$, namely

$$
\left|E_{\lambda}(\hat{\boldsymbol{a}}, \hat{\boldsymbol{b}})-E_{\lambda}(\hat{\boldsymbol{a}}, \hat{\boldsymbol{c}})\right| \leq 1+E_{\lambda}(\hat{\boldsymbol{b}}, \hat{\boldsymbol{c}}) .
$$

The observation by Bell is that these inequalities are violated by the quantum prediction (3) for particular directions $\hat{\boldsymbol{a}}, \hat{\boldsymbol{b}}, \hat{\boldsymbol{c}}$ (for instance differing by $\frac{\pi}{8}$ ), hence offering the possibility to discriminate between standard quantum mechanics and hidden variables experimentally. Such experiments were realized with photon polarizations by Aspect et al. [12] and displayed a clear disagreement with inequality (4) while confirming the quantum prediction (3).

This result somehow signs the death warrant of hidden variables characterizing the microscopic system (in this case the pair of particles). Let us now show that in contrast it does not prevent the hypothesis of hidden variables characterizing the internal state of the measurement apparatus. In the present situation, we introduce variables $\Lambda_{1}$ and $\Lambda_{2}$ that correspond to the apparatus measuring particle 1 and 2 respectively. In the same spirit as Bell, we do not attempt to find the explicit physical nature of these variables; we only assume that they exist and that they determine the experimentally observed results, together with the particle state and the apparatus orientation.

Let us denote by $R(|\sigma\rangle, \hat{\boldsymbol{a}}, \Lambda)=\frac{2}{\hbar} \boldsymbol{s} \cdot \hat{\boldsymbol{a}}$ the result of the measurement of the projection of the spin $s$ of one of the spin $\frac{1}{2}$ particles assumed to be in state $|\sigma\rangle$, in one of the measurement apparatus oriented along direction $\hat{\boldsymbol{a}}$. This result is assumed to be determined by the value of the hidden variable $\Lambda$ characterizing the internal state of the apparatus. The precise value of $\Lambda$ is not known but its probability distribution $p(\Lambda)$ has to satisfy $\sum_{\Lambda} p(\Lambda)=1$, where the sum runs over all the possible values of $\Lambda$. The two apparatuses are assumed to be identical; a unique function $R$ hence describes both of them.

This function satisfies the following conditions to describe the measurement process correctly:

1. it takes the values \pm 1 as the particle has spin $\frac{1}{2}$;

2. the measurement of a polarized state made along its polarization direction is reproducible, whatever the internal state of the apparatus; hence $R\left(| \pm\rangle_{\hat{a}}, \hat{\boldsymbol{a}}, \Lambda\right)= \pm 1, \forall \Lambda$;

3. the mean value of a state polarized along direction $\hat{\boldsymbol{a}}$ measured through an apparatus oriented along direction $\hat{\boldsymbol{b}}$ is related to the angle between $\hat{\boldsymbol{a}}$ and $\hat{\boldsymbol{b}}$ as

$$
\sum_{\Lambda} p(\Lambda) R\left(|+\rangle_{\hat{a}}, \hat{\boldsymbol{b}}, \Lambda\right)=\hat{\boldsymbol{a}} \cdot \hat{\boldsymbol{b}}
$$


which also implies that

$$
\sum_{\Lambda} p(\Lambda) R\left(|-\rangle_{\hat{\boldsymbol{a}}}, \hat{\boldsymbol{b}}, \Lambda\right)=-\hat{\boldsymbol{a}} \cdot \hat{\boldsymbol{b}}
$$

Within these hypotheses, the mean correlation between the two measurements reads

$$
E_{\Lambda_{1} \Lambda_{2}}(\hat{\boldsymbol{a}}, \hat{\boldsymbol{b}})=\sum_{\Lambda_{1} \Lambda_{2}} p\left(\Lambda_{1}\right) p\left(\Lambda_{2}\right) R\left(\left|\sigma_{1}\right\rangle, \hat{\boldsymbol{a}}, \Lambda_{1}\right) R\left(\left|\sigma_{2}\right\rangle, \hat{\boldsymbol{b}}, \Lambda_{2}\right)
$$

where $\left|\sigma_{i}\right\rangle$ is the state of particle $i$ as measured by apparatus $i$. To fix ideas, we assume that the measurement in apparatus 1 , oriented along direction $\hat{\boldsymbol{a}}$, is made before that in apparatus 2 , oriented along direction $\hat{\boldsymbol{b}}$ (but the time interval between both measurements can be made arbitrarily small). Hence we choose to write the singlet state (1) with $\hat{\boldsymbol{u}}=\hat{\boldsymbol{a}}$. The state of particle 1 , as seen by apparatus 1 , is thus $\left|\sigma_{1}\right\rangle=\frac{1}{\sqrt{2}}\left[|+\rangle_{\hat{a}}-|-\rangle_{\hat{a}}\right]$, which implies that the first measurement gives +1 or -1 in $50 \%$ of the cases. This allows an easy calculation of the first sum in (7). Immediately after this first measurement, particle 2 is put in either a down or an up state along direction $\hat{\boldsymbol{a}}$, depending on the result obtained for the first measurement. The second apparatus being oriented along direction $\hat{b}$, the second sum in (7) is calculated using (5) and (6). One has finally

$$
\begin{aligned}
E_{\Lambda_{1} \Lambda_{2}}(\hat{\boldsymbol{a}}, \hat{\boldsymbol{b}}) & =\frac{1}{2} \sum_{\Lambda_{2}} p\left(\Lambda_{2}\right)\left[R\left(|-\rangle_{\hat{\boldsymbol{a}}}, \hat{\boldsymbol{b}}, \Lambda_{2}\right)-R\left(|+\rangle_{\hat{\boldsymbol{a}}}, \hat{\boldsymbol{b}}, \Lambda_{2}\right)\right] \\
& =\frac{1}{2}(-\hat{\boldsymbol{a}} \cdot \hat{\boldsymbol{b}}-\hat{\boldsymbol{a}} \cdot \hat{\boldsymbol{b}})=-\hat{\boldsymbol{a}} \cdot \hat{\boldsymbol{b}} .
\end{aligned}
$$

The result is thus identical to the standard quantum mechanics prediction (3). Apparatus hidden variables hence also lead to a violation of Bell's inequalities (4), in agreement with experiment.

While still agreeing with standard quantum mechanics, the present theory is deterministic, as the measurement results are determined by the actual values of $\Lambda_{1}$ and $\Lambda_{2}$. Another advantage is that these hidden variables are local, in the sense that $\Lambda_{1}$ only characterizes the state of apparatus 1 and $\Lambda_{2}$ only characterizes the state of apparatus 2 . Hence there is no need to consider generalized inequalities for non-local hidden variables [21]. The price to pay for this simplicity is a strong nonlocality, the consequences of which will be discussed in a relativistic perspective in the speculative conclusions. Before that, we would like to consider a model of measurement apparatus in which the physical nature of these internal degrees of freedom can be explicitly described, to test the hypothesis that they determine the measurement outcome. To do that, we consider what is probably the simplest possible measurement apparatus to model schematically, namely the cloud chamber mentioned in the introduction. There the $\Lambda$ internal variables could just be the positions of the atoms in the chamber. Note that a schematic model of Stern-Gerlach apparatus could be built on the same basis, with the screen being replaced by a cloud chamber. As for photon polarization measurement apparatuses, a microscopic model should be based on internal electronic degrees of freedom; mesoscopic or cold polarisers might be interesting to consider in that respect.

\section{A schematic model for deterministic quantum measurement}

In the present section, we revisit an idea proposed by Mott [2] and discussed by Bell [22], namely the measurement of a spherical-wave $\alpha$-particle state in a Wilson cloud chamber. Mott shows that the observation of linear tracks can be explained by the interaction of the $\alpha$-particle spherical wave with the atoms of the gas filling the chamber. Here, we reproduce his calculation but interpret his result differently: we show that the presence of the gas might not only explain the appearance of linear tracks 
(a)

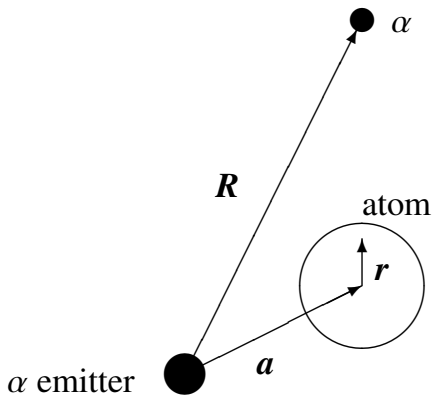

(b)

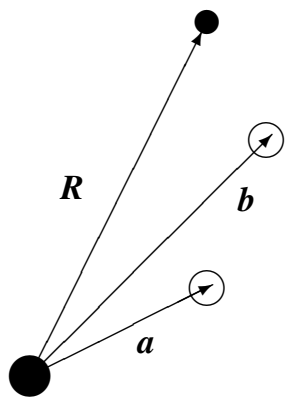

Figure 1. Theoretical systems used to describe the emission of an $\alpha$ particle by an $\alpha$ emitter in a cloud chamber. Nuclei are represented as filled circles; cloud-chamber atoms are represented as empty circles. Only (a) the atom (b) the two atoms nearest to the $\alpha$ emitter are considered.

but also determine which linear track is actually observed for a given microscopic configuration of the gas atoms. Mott's model could thus be considered as the first model for a deterministic decoherent process of wave-function reduction taking place during a quantum measurement.

Let us first consider the interaction between an $\alpha s$-wave emitted by a radioactive nucleus located at the origin and a single atom in a cloud chamber [see figure 1(a)]. We assume that this atom, which is the first obstacle seen by the $\alpha$ wave, is immobile at a fixed position $\boldsymbol{a}$. This is justified as the typical thermal agitation energy of molecules in a cloud chamber ( $\mathrm{a}$ few $\mathrm{meV}$ ) is much lower than the typical $\alpha$-particle kinetic energy $E_{\alpha}(\mathrm{a}$ few $\mathrm{MeV})$. This obstacle is rather generic; its precise nature will not affect the general conclusions drawn below. To simplify calculations, we assume that it is described by an internal Hamiltonian $H$ with internal variables $r$ and we only consider two excitations levels: its ground state $E_{0}$ and its first excited state $E_{1}$. The corresponding wave functions $\psi_{0}(\boldsymbol{r})$ and $\psi_{1}(\boldsymbol{r})$ are orthonormal, i.e. $\left\langle\psi_{i} \mid \psi_{j}\right\rangle=\delta_{i j}$ for $i, j=0,1$; they are assumed to be localised in a region of size $s$, e.g. the typical size of an atom. The total hamiltonian of the system is thus

$$
H=-\frac{\hbar^{2}}{2 m_{\alpha}} \Delta_{\boldsymbol{R}}+H+V(\boldsymbol{R}, \boldsymbol{r}),
$$

where $m_{\alpha}$ is the $\alpha$-particle mass, $\boldsymbol{R}$ its position and $V(\boldsymbol{R}, \boldsymbol{r})$ describes its interaction with the obstacle. In the following, this interaction is assumed to be small, which allows the use of a Born-expansion perturbative treatment [23].

Within these hypotheses, the stationary wave function of the total $\alpha$-particle + obstacle system can be approximately described as a first-order Born-expanded coupled-channel wave function,

$$
\Psi(\boldsymbol{R}, \boldsymbol{r}) \approx f_{0}^{(0)}(\boldsymbol{R}) \psi_{0}(\boldsymbol{r})+f_{0}^{(1)}(\boldsymbol{R}) \psi_{0}(\boldsymbol{r})+f_{1}^{(1)}(\boldsymbol{R}) \psi_{1}(\boldsymbol{r}) .
$$

In this equation, the superscript refers to the order in the Born expansion while the subscript refers to the excitation state of the obstacle. The first term corresponds to the situation where the $\alpha$ particle is unaffected by the presence of the obstacle; its wave function is thus an outgoing spherical wave of kinetic energy $E_{\alpha}$ and wave number $k$,

$$
f_{0}^{(0)}(\boldsymbol{R})=\frac{e^{i k R}}{R}, \quad k=\sqrt{2 m_{\alpha} E_{\alpha} / \hbar^{2}} .
$$


(a)

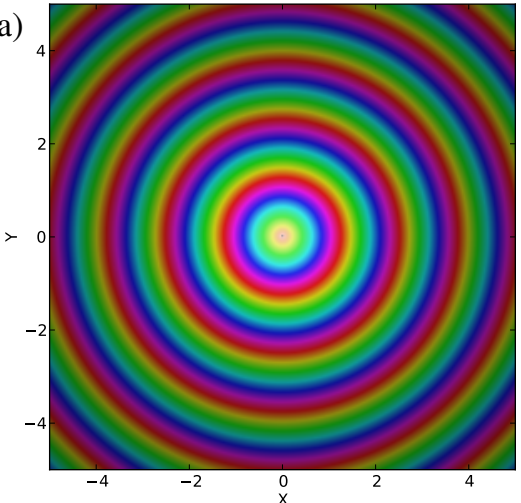

(b)

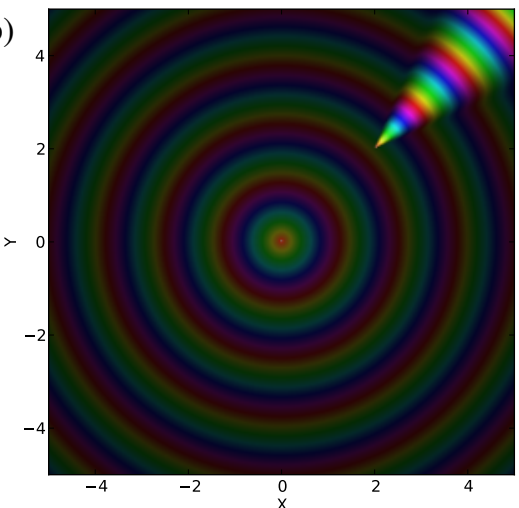

Figure 2. $\alpha$-particle wave function (a) in the absence (b) in the presence of an obstacle. In case (b), only the elastic-scattering part of the wave function is represented and the wave function is assumed to have reached its first-order Born-approximation asymptotic behaviour everywhere. The phase of the wave functions is represented by the colour hue while their modulus is represented by the colour brightness [24].

This wave function is represented in figure 2(a), where the exponential phase factor is visible as coloured rings, while the $1 / R$ modulus necessary for flux conservation is visible as decreasing brightness. The second term of (10) is the first-order Born expansion of the elastic scattering; the wave function $f_{0}^{(1)}(\boldsymbol{R})$ hence corresponds to the same kinetic energy $E_{\alpha}$ as the zeroth-order term. Note that this term was not considered by Mott [2]. The third term of (10) corresponds to the excitation of the obstacle by the $\alpha$ particle; hence, by energy conservation, the wave function $f_{1}^{(1)}(\boldsymbol{R})$ is characterized by an energy $E_{\alpha}^{\prime}=E_{\alpha}+E_{0}-E_{1} \equiv \frac{\hbar^{2} k^{\prime 2}}{2 m_{\alpha}}$ and wave number $k^{\prime}$.

These first-order terms can be calculated with the help of the Green-function method (see [1]) and are expressed in terms of matrix elements of potential $V$,

$$
V_{j 0}(\boldsymbol{R}) \equiv \int d \boldsymbol{r} \psi_{j}^{*}(\boldsymbol{r}) V(\boldsymbol{R}, \boldsymbol{r}) \psi_{0}(\boldsymbol{r}), \quad j=0,1 .
$$

The remarkable feature of functions $f_{j}^{(1)}(\boldsymbol{R})$, as already stressed by Mott [2], is that they present an asymptotic behaviour strongly peaked along direction $\hat{\boldsymbol{a}}$. To show that, we first assume that the energy excitation of the obstacle is negligible with respect to the $\alpha$-particle kinetic energy, which implies $k^{\prime} \approx k$. Then, by defining the unit vector $\hat{\boldsymbol{e}} \equiv \frac{\boldsymbol{R}-\boldsymbol{a}}{|\boldsymbol{R}-\boldsymbol{a}|}$ and the angle $\theta$ between $\hat{\boldsymbol{a}}$ and $\hat{\boldsymbol{e}}, \theta=\arccos (\hat{\boldsymbol{a}} \cdot \hat{\boldsymbol{e}})$, one gets the asymptotic behaviour

$$
f_{j}^{(1)}(\boldsymbol{R}) \underset{a,|\boldsymbol{R}-\boldsymbol{a}| \gg s}{\approx} \frac{e^{i k|\boldsymbol{R}-\boldsymbol{a}|}}{|\boldsymbol{R}-\boldsymbol{a}|} I_{j}(\theta) .
$$

That is a spherical wave emitted in $\boldsymbol{a}$, modulated by an angular-dependent function

$$
I_{j}(\theta)=\frac{m_{\alpha}}{2 \pi \hbar^{2}} \frac{e^{i k a}}{a} \int d \boldsymbol{R}^{\prime} V_{j 0}\left(\boldsymbol{R}^{\prime}\right) e^{i \boldsymbol{q} \cdot \boldsymbol{R}^{\prime}},
$$

where $\boldsymbol{q} \equiv k(\hat{\boldsymbol{a}}-\hat{\boldsymbol{e}})$, is the transferred momentum related to $\theta$ by $q=2 k \sin \frac{\theta}{2}$. To establish (13) and (14), we have used the fact that matrix elements (12) only have a non negligible value in a volume of 
size $s$ around the obstacle position $\boldsymbol{a}$. Expression (14) contains the Fourier transform of these matrix elements, a well-known feature of the first-order Born approximation. These matrix elements can be calculated explicitly for various assumptions on the obstacle, in particular in the case of hydrogen-like wave functions $[2,23]$. Here, as we are rather interested in general properties of the scattering wave function (10), we simply assume that the matrix elements (12) have a spherical Gaussian form factor of width $s$. This allows us to plot a typical behaviour of the wave function in panel (b) of figure 2 . In this figure, only the spherical wave and the peaked elastic-scattering waves are shown; the inelasticscattering wave for $k^{\prime}<k$ would have a behaviour similar to the elastic-scattering one but with a broader peak.

Let us now come to the delicate point of the wave-function normalization, which is not discussed by Mott [2] either. Here, we fix this normalization by imposing that the total probability flux $F$ across a sphere centred on the $\alpha$ emitter and large enough to include the obstacle should be the same whether this obstacle is present or not. This seems natural, as the activity of the radioactive source should be independent of its surroundings, but we shall show that it has important physical consequences. Without obstacle, this probability flux takes the value

$$
F_{\text {without }}=4 \pi \frac{\hbar k}{m_{\alpha}} \equiv 4 \pi v_{\alpha}
$$

which only depends on the $\alpha$-particle velocity $v_{\alpha}$. With an obstacle, the calculation leads to a larger flux because of the last two terms of (10). Hence for the fluxes to be equal with and without obstacle the coupled-channel wave function (10) has to be multiplied by a factor $|C|$ smaller than 1 and defined by

$$
\frac{1}{|C|^{2}}=1+\frac{1}{2} \int_{0}^{\pi} d \theta \sin \theta\left|I_{0}(\theta)\right|^{2}+\frac{1}{2} \frac{v_{\alpha}^{\prime}}{v_{\alpha}} \int_{0}^{\pi} d \theta \sin \theta\left|I_{1}(\theta)\right|^{2},
$$

where $v_{\alpha}^{\prime}$ is the $\alpha$-particle velocity in the inelastic case. The flux associated with the spherical wave thus reads

$$
F_{\text {spherical }}=|C|^{2} F_{\text {without }}, \quad|C|<1,
$$

in this case, which can be interpreted as the first step of the $\alpha$-particle spherical-wave reduction due to the presence of the gas-chamber atoms. This key result is shown in figure 2(b), where the brightness of the spherical wave is reduced.

Following Mott [2], we next consider the scattering on two successive obstacles located in $\boldsymbol{a}$ and $\boldsymbol{b}$ [see figure 1(b)]. A second-order Born expansion is required and the very important result by Mott is that the second-order wave function $f_{11}^{(2)}$, corresponding to an excitation of both atoms, is significantly different from zero only if $\boldsymbol{a}$ and $\boldsymbol{b}$ are aligned (for atoms $\boldsymbol{a}$ and $\boldsymbol{b}$ to be excited, atom $\boldsymbol{b}$ has to lie in the narrow cone generated by the presence of atom $\boldsymbol{a}$ ). This explains the appearance of linear tracks from an initially symmetric spherical wave function. Here, we complete this result by noting that, in case of an alignment, the wave function should be multiplied by a factor of the order of $|C|^{2}$ for the probability fluxes to be equal with or without obstacles. For $N$ atoms aligned with the $\alpha$ emitter, this reduction factor should be of the order of $|C|^{N}$, which implies that the spherical wave tends to zero if $N$ is large enough. We conjecture this might be a model for the phenomenon of wave-function reduction. In the case of a cloud chamber consisting of randomly-distributed atoms, this mechanism would select the direction of the atoms best-aligned with the $\alpha$ emitter: only for these atoms would the high-order component of the wave function not vanish. The wave-function reduction would thus only occur if some atoms are aligned in a given direction; that direction would be the measured linear track. This model would thus provide a deterministic explanation to the apparent randomness of quantum measurement. 


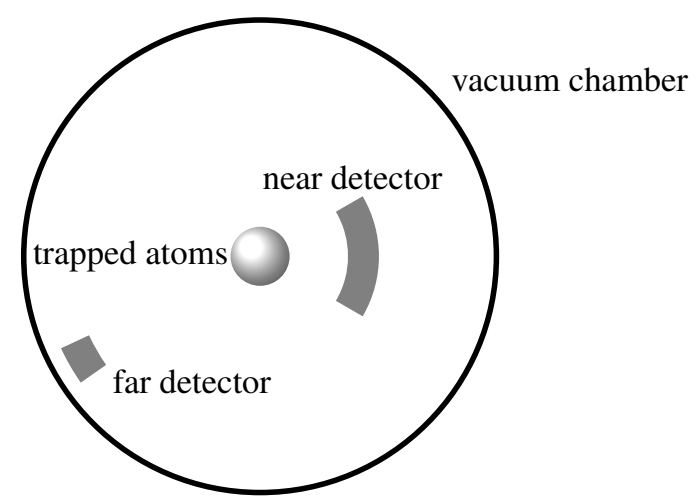

Figure 3. Schematic experimental set-up for the detection by a far detector of the reduction of $s$ or $p$ waves emitted by trapped atoms, in the presence of a detector close to the emitter.

One should keep in mind that the above reasoning rests on simplifying hypotheses, which should be tested carefully before final conclusions. First, the calculation is based on the Born approximation, which violates unitarity [23], and on a truncated coupled-channel approximation. Hence, convergence tests of these approximations or a direct numerical solution of the stationary Schrödinger equation should be performed. Second, our interpretation is based on a stationary state calculation, whereas it has a temporal content in essence: the wave function is first affected by the atom nearest to the source, then by the second nearest atom and so on. This stationary-state approach is an approximation of the full solution of the time-dependent Schrödinger equation. There again, solving this equation numerically for instance for an initial spherical wave packet would provide very useful checks. Nevertheless, assuming that the reduction of the spherical wave in the presence of an obstacle shown in figure 2 is real, it could be detected experimentally. A possible set-up is illustrated in figure 3 and discussed in [1]. The trapped atoms (or ions) could either have an $s$-wave $\alpha$ emitter as nucleus or be electric-dipole-photon emitters themselves.

\section{Speculative conclusions: faster-than-light or not?}

As a conclusion we propose an interpretation of quantum mechanics which is deterministic in essence. The outcome of a decoherent process like a measurement can in principle be predicted from the knowledge of the microscopic state of the environment or macroscopic apparatus. This state being inaccessible in most practical situations, the outcomes of decoherence processes generally seem random. Nevertheless this randomness now appears as much less fundamental and unavoidable than in standard quantum mechanics presentations. We have first shown, without making any attempt to describe microscopic states explicitly, that this interpretation leads to a violation of Bell's inequalities, despite the fact that this microscopic state can be seen as a hidden variable. Next we have studied explicitly a simplified model of measurement apparatus, the cloud chamber, and obtained preliminary results that support our interpretation: the wave-function collapse leading to the measurement outcome could be determined by the positions of the atoms in the cloud chamber. Let us stress that this interpretation, to put it in Mott's words [2], is based on "wave mechanics unaided": no further ingredient (pilot wave, many worlds, free will...) than wave functions is required.

Let us next notice that both types of quantum states considered above are highly non local. The EPR intricated Bell state (1) implies a perfect and instantaneous correlation of the measured states of 
particles 1 and 2, despite the fact that these states are not known in advance. Similarly an $\alpha$-particle spherical state implies that the detection of the particle in one direction immediately prevents the particle from being detected in other directions. These states themselves do not violate special relativity and causality as they can be explained by their unique spatio-temporal origin either at the creation of the pair or at the emission of the $\alpha$-particle. When submitted to a measurement process their non locality is revealed and an action at a distance is necessary to explain the perfect correlations between space-separated events. In usual interpretations of quantum mechanics, causality is however preserved by the random character of the measurement results [13], as already mentioned in the introduction.

In contrast, in the present interpretation this random character is replaced by a deterministic interpretation. For the spherical wave, the presence of the obstacle in a given direction might immediately lead to a reduction of the spherical wave in all other directions, an effect which could be tested experimentally [1]. For the EPR pair, the state of apparatus 1, formally described by the value of $\Lambda_{1}$, determines the result of the measurement performed on particle 1. But particles 1 and 2 being fully correlated, this measurement is actually a measurement of the particle pair as a whole: it immediately affects the state of both particles by reducing their wave function to either $|+\rangle_{\hat{a} 1}|-\rangle_{\hat{a} 2}$ or $|-\rangle_{\hat{a} 1}|+\rangle_{\hat{a} 2}$. The value of $\Lambda_{1}$ thus also instantaneously determines the state of particle 2, even though apparatus 1 and particle 2 can be separated by very large distances (or by very long optical fibers). It is the very structure of the intricated state (1) which allows this actual "spooky action at a distance". Hence, the present interpretation suggests that quantum non locality allows faster-than-light information transfer, provided the internal state of a measurement-apparatus-like system can be controlled (mesoscopic cloud chambers or polarizers could be helpful with that respect).

This raises of course special-relativity paradoxes, which might be considered as a very strong "no-go" argument for our interpretation. We rather speculate that our approach might help settle the long-standing conflict between special relativity and quantum mechanics, Einstein's two warring daughters. This conflict was made explicit by Einstein himself in the EPR paper, then brought to a climax by Bell and his inequalities, to finally burst into a triumphant victory of quantum mechanics in Aspect's famous experiments. We believe the quantum world to be fully deterministic and non local and hope this work will help testing this very strong hypothesis thoroughly.

\section{Acknowledgements}

JMS acknowledges very interesting discussions with several colleagues at different stages of this work, in particular with N. J. Cerf, D. Baye, J. Barbour, P. Gaspard, C. Semay, P. Capel and F. Binon.

\section{References}

[1] J.-M. Sparenberg, R. Nour and A. Manço (2012), arXiv:quant-ph/1212.2256v1

[2] N.F. Mott, Proc. R. Soc. A126, 79 (1929); also in N.F. Mott, A.S. Alexandrov, eds., Sir Nevill Mott, 65 years in physics (World Scientific, Singapore, 1995)

[3] M. Born, ed., The Born-Einstein letters (Walker, New York, 1971), translated by I. Born

[4] J. Barbour, The end of time: the next revolution in physics (Oxford University, New York, 2001)

[5] A. Einstein, B. Podolsky, N. Rosen, Phys. Rev. 47, 777 (1935)

[6] J.S. Bell, Physics 1, 195 (1964); also in [25]

[7] D. Bohm, Y. Aharonov, Phys. Rev. 108, 1070 (1957)

[8] J.S. Bell, Rev. Mod. Phys. 38, 447 (1966); also in [25]

[9] J.F. Clauser, M.A. Horne, A. Shimony, R.A. Holt, Phys. Rev. Lett. 23, 880 (1969) 
[10] S.J. Freedman, J.F. Clauser, Phys. Rev. Lett. 28, 938 (1972)

[11] A. Aspect, P. Grangier, G. Roger, Phys. Rev. Lett. 47, 460 (1981)

[12] A. Aspect, J. Dalibard, G. Roger, Phys. Rev. Lett. 49, 1804 (1982)

[13] P. Eberhard, Nuov. Cim. B 46, 392 (1978)

[14] P. Gaspard, Mécanique quantique, Quantum physics lecture notes (unpublished), Université libre de Bruxelles (2001)

[15] W.H. Zurek, Phys. Rev. D 24, 1516 (1981)

[16] R. Omnès, Rev. Mod. Phys. 64, 339 (1992)

[17] W.H. Zurek, Phys. Today 44, 36 (1991); Los Alamos Science 27, 2 (2002)

[18] N.G. van Kampen, Physica A 153, 97 (1988)

[19] P. Gaspard, M. Nagaoka, J. Chem. Phys. 111, 5676 (1999)

[20] J.V. Corbett, D. Home (2008), arXiv:quant-ph/0802.2443

[21] A.J. Leggett, Found. Phys. 33, 1469 (2003)

[22] J.S. Bell, Quantum mechanics for cosmologists, in Quantum Gravity 2, edited by C. Isham, R. Penrose, D. Sciama (Clarendon, Oxford, 1981), pp. 611-637; also in [25]

[23] J.R. Taylor, Scattering Theory: The Quantum Theory on Nonrelativistic Collisions (Wiley, New York, 1972)

[24] B. Thaller, Visual quantum mechanics (Springer, New York, 2000)

[25] J.S. Bell, Speakable and unspeakable in quantum mechanics, 2nd edn. (Cambridge University, New York, 2001) 